\title{
The position of the media as a tool of communication and education on severe weather in Malaysia
}

\begin{abstract}
Information by the media in advance of severe weather occurrences can prepare the public to respond properly during an emergency situation. At the same time, the media should work as a channel to communicate and promote proper public response to severe weather threats. The objective of this study is to examine the position of the media as a tool of communication and education on severe weather in Malaysia. Results indicate that the present role of the media in Malaysia is still insufficient in communicating and educating the public on severe weather.
\end{abstract}

Keyword: Severe weather; The media; Communication; Education 